\title{
Diagnostic accuracy of computed tomography to identify adenomas among adrenal incidentalomas in an endocrinological population
}

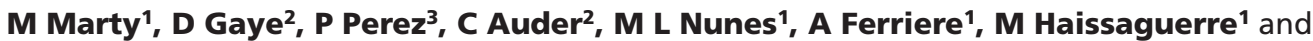 \\ A Tabarin ${ }^{1}$
}

Departments of ${ }^{1}$ Endocrinology, Diabetes and Nutrition, ${ }^{2}$ Radiology, CHU Haut-Leveque and University of Bordeaux, Bordeaux, France, and ${ }^{3}$ Public Health Department, Clinical Epidemiology Unit INSERM, CIC 1401 and Bordeaux University Hospital, Bordeaux, France
Correspondance should be addressed to A Tabarin

Email

antoine.tabarin@chubordeaux.fr

\section{Abstract}

Context: The recent recommendations of the European Endocrine Society states that the performance of computed tomography (CT) to characterize 'true' adrenal incidentalomas (Als) remains debatable.

Objective: To determine relevant thresholds for usual CT parameters for the diagnosis of benign tumors using robust reference standard among a large series of 'true' Als recruited in an endocrinological setting.

Design: Retrospective study of 253 Als in 233 consecutive patients explored in a single university hospital: 183 adenomas, 33 pheochromocytomas, 23 adrenocortical carcinomas, 5 other malignant tumors and 9 other benign tumors. Reference standard was histopathology in $118 \mathrm{Als}$, biological diagnosis of pheochromocytoma in 2 Als and size stability after at least 1 year of follow-up in 133 Als.

Methods: Sensitivity, specificity and positive and negative predictive values were estimated for various thresholds of size, unenhanced attenuation (UA), relative and absolute wash-out (RPW, APW) of contrast media. 197 scans were reviewed independently in a blinded fashion by two expert radiologists to assess inter-observer reproducibility of measurements.

Results: Criteria associated with a $100 \%$ positive predictive value for the diagnosis of benign Al were: a combination of size and UA: $30 \mathrm{~mm}$ and $20 \mathrm{HU}$ or $40 \mathrm{~mm}$ and $15 \mathrm{HU}$, respectively; RPW $>53 \%$; and APW $>78 \%$. Non-adenomatous Als with rapid contrast wash-out were exclusively benign pseudocysts and pheochromocytomas, suggesting that classical thresholds of $60 \%$ and $40 \%$ for APW and RPW, respectively, can be safely used for patients with normal metanephrine values. Inter-observer reproducibility of all parameters was excellent (intra-class correlation coefficients: 0.96-0.99).

Conclusions: Our study, the largest conducted in Als recruited in an endocrinological setting, suggests safe thresholds for quantitative CT parameters to avoid false diagnoses of benignity.

\section{Introduction}

Adrenal incidentalomas (AIs) are discovered by chance in around $5 \%$ of patients who undergo computed tomography (CT) for indications other than adrenal
European Journal of masses. A number of etiologies can give rise to AIs, but it is crucial to determine whether or not a given $\mathrm{AI}$ is malignant or responsible for hormonal hypersecretion, 
two situations in which surgical excision is recommended (1). The prevalence of malignant and hormonally active AIs is largely dependent on the patient referral pattern (2, $3,4)$. In addition to laboratory studies, CT is a cornerstone investigation for etiological diagnosis of AIs and more specifically for the identification of adrenal adenomas. Size, unenhanced spontaneous attenuation (UA) and contrast medium wash-out have been the most widely used radiological criteria $(3,4,5)$.

However, as emphasized by the recent recommendations of the European Endocrine Society (1) and by the authors of a joint comprehensive analysis of the literature (3), most studies evaluating the accuracy of CT carried a high risk of bias and were ineligible for metaanalysis because of multiple caveats (lack of an appropriate reference standard, small size of series, poor reporting of the indications for imaging, etc.). Indeed, more than $90 \%$ of retrieved papers had to be excluded. It is also noteworthy that less than one-fifth of published studies included a majority of participants with purely incidental findings (3). Interestingly, another meta-analysis of large studies including mostly unselected patients with AIs detected in situations similar to the clinical scenario of patients referred to an endocrinologist (e.g. exclusion of surgical series, histopathological series and oncological series) showed a very low prevalence of carcinomatous and potentially life-threatening hormonally active tumors while benign, non-functional lesions represented more than $80 \%$ of cases (2). Therefore, the recruitment bias pointed by Dinnes and coworkers in their meta-analysis of imaging studies in AIs (3) may have enriched the series in cases of metastasis and life-threatening lesions, by comparison with a standard endocrinological population. Elsewhere, the paucity of incidentally discovered ACCs in this metanalysis, which, although rare, represent the main etiology of malignant AIs in the endocrinological setting, was small, impeding precise evaluation of their CT characteristics. The rare series that have analyzed the CT characteristics of these neoplasms do not mention if incidentally discovered ACCs display the same imaging characteristics as other ACCs $(1,6,7,8,9)$. This question is important, as recommended laboratory investigations are likely to be poorly informative, as it can be hypothesized that most incidentally discovered ACCs will be hormonally silent (10). Together, these methodological caveats may limit and bias our assessment of the accuracy of CT in the evaluation of AIs. Consequently, the recommendations of the European Endocrine Society emphasize the need for methodologically adequate complementary studies to define CT performance, and pertinent thresholds, including contrast-enhanced timed wash-out CT criteria, to distinguish benign from malignant AIs (1).

The purpose of this study was to reassess the performance of CT and determine safe thresholds for the diagnosis of adenomas and benign tumors among 'true' AIs, using appropriate histological and imagingbased follow-up reference standard in the largest cohort of consecutively referred patients in an endocrinological setting.

\section{Patients and methods}

We reviewed the charts of 417 consecutive patients referred to our endocrinology department for the evaluation of AIs measuring at least $10 \mathrm{~mm}$ between January 2000 and December 2013 (Fig. 1).

In addition to CT, all the patients underwent detailed clinical and endocrine investigations, including at least the $1 \mathrm{mg}$ dexamethasone suppression test, 24-h urinary metanephrines excretion or plasma metanephrines and plasma aldosterone and renin in most of hypertensive patients. The data were collected under conditions of regular clinical care, and local ethics committee approval was obtained for their analysis.

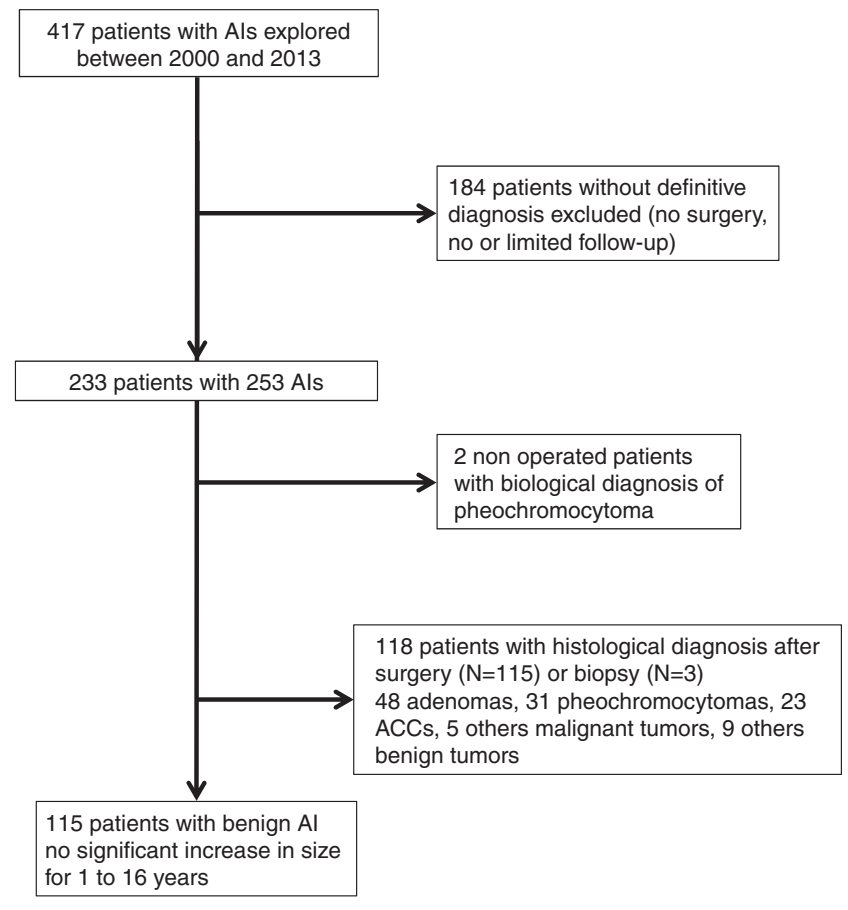

Figure 1

Flow diagram of patients with adrenal incidentalomas. 
The reference standard diagnosis was established by histopathological analysis after surgical excision $(N=115)$ or percutaneous biopsy $(N=3)$, in front of clearly elevated urinary metanephrines in 2 patients with suspected unilateral pheochromocytomas who declined surgery or because of size stability after at least one year of follow-up (median 3.0 years, range $=1-16$ years) in patients with normal laboratory results $(N=113)$. This last condition in which histological results were lacking was arbitrarily defined as 'adenoma'. One hundred eighty-four patients with apparently benign AIs on CT using classical criteria in a vast majority $(4,11)$ but who did not undergo surgery or receive adequate follow-up were excluded from the study. The study cohort therefore consisted of 233 patients (93 men and 140 women, age $56.4 \pm 10.5$ years) harboring 253 AIs. The AIs were located on the left side in 151 cases and on the right in 102 cases. CT was indicated for various symptoms (mainly abdominal pain) but was never prescribed for the purpose of imaging the adrenal glands or for follow-up/staging of neoplasms. At presentation, $10.7 \%$ of patients had a history of cancer that was considered to be in longlasting remission (breast $(n=7)$, spin and basocellular cutaneous cancers $(n=4)$, colon $(n=3)$, thyroid $(n=3)$, chronic lymphocytic leukemia $(n=2)$, uterus $(n=1)$, ovarian tumor $(n=1)$ and contralateral adrenocortical carcinoma $(n=1)) .115$ of the 233 patients underwent surgical excision and 3 underwent biopsy of the AI, due to suspicion of malignancy $(n=33)$, indeterminate imaging features $(n=19)$, large size $(n=4)$ or hormone production $(n=62)$. The definitive etiological diagnosis was adenoma for 183 AIs (72\%). Forty-eight adenomas (26\%) were diagnosed on histopathological analysis and 135 (74\%) based on the follow-up criterion. The remaining 70 AIs consisted of 33 pheochromocytomas (13.0\% of the study cohort), 23 adrenocortical carcinomas (ACC) $(9.1 \%$ of the study cohort), 5 malignant tumors (1 metastasis, 1 angiosarcoma, 1 leiomyosarcoma, 1 bilateral lymphoma) and 9 other tumors (2 ganglioneuromas, 2 schwannomas, 2 pseudocysts, 1 hematoma, 1 atypical myelolipoma, 1 hemangioma).

\section{CT methods}

Multidetector CT images were obtained with either a 64-section (Somatom Definition; Siemens) or a 16-section device (Sensation; Siemens) with the following technical parameters: pitch $0.8-1$, collimation $24 \times 1.2 \mathrm{~mm}$ or $16 \times 1.5 \mathrm{~mm}$, slice thickness $2 \mathrm{~mm}$; variable tube current ( $180 \mathrm{~mA}$ reference depending on patient size) and $120 \mathrm{kVp}$.
Unenhanced images were obtained first, followed by dynamic contrast images $60 \mathrm{~s}$ (early contrast-enhanced) and $10 \mathrm{~min}$ (delayed contrast-enhanced) after bolus administration of an intravenous contrast agent. The bolus consisted of up to $2 \mathrm{~mL} / \mathrm{kg}$ with an iodine concentration of $300 \mathrm{mg} / \mathrm{mL}$ at a flow rate of $2 \mathrm{~mL} / \mathrm{s}$.

Lesion size was calculated by using the AI slice with the largest surface area. Average attenuation values were measured with circular or ovoid region of interest (ROI) cursors on the unenhanced, contrast-enhanced and delayed contrast-enhanced images. The ROI was placed over the AI including one-half to two-thirds the area of the lesion except edges to minimize partial volume effects. Cystic, calcified necrotic and hemorrhagic areas were excluded from the analysis.

Contrast wash-out was studied in 82 patients with UA valuesnottypicalofabenignadenoma ( $>10$ Hunsfieldunits). From the attenuation values recorded on the unenhanced, dynamic and delayed CTs, the APW and RPW values were calculated as follows: $\mathrm{APW}=($ enhanced - delayed $) /$ (enhanced-unenhanced) $\times 100$; RPW $=$ (enhanced-delayed) $/$ enhanced $\times 100$. All attenuation values are shown in Hounsfield units. In the presence of large and heterogeneous AIs, we selected during portal venous phase acquisitions the CT slice in which the lesion was the most homogenous with the largest tissular portion, to define the ROI. Attenuation values during each phase of the wash-out study were then measured in the same area of the lesion, at exactly the same level of CT image (matched slices).

To analyze the inter-observer reproducibility of CT measurements (size, UA, RPW and APW), 197 scans were reviewed independently by two expert radiologists who were blinded to the clinical, pathological and follow-up data and the definitive diagnosis.

\section{Statistical analysis}

Unless otherwise specified, data are means \pm s.D. The target condition was defined as any etiological diagnosis that would not necessitate further imaging exploration i.e. adenomas and other benign tumors. The diagnostic accuracy of the size, UA, RPW and APW were estimated by comparison with the reference standard. When using a threshold, these quantitative parameters were defined as positive when taking lower values for size and UA and higher values for RPW and APW. Sensitivity was defined as the probability to have a positive test in patients with the target condition. Specificity was defined as the probability to have a negative test in patients without 
the target condition. Confidence intervals (95\% CI) were calculated by using the exact binomial distribution for proportions. A receiver-operating characteristics (ROC) curve was drawn for each CT parameter. Areas under the curve (AUC) and their confidence intervals were estimated by using the \% ROC macro (SAS Institute). Agreement between values for a given CT parameter obtained by the two radiologists was assessed by calculating the intra-class correlation coefficient and confidence interval.

For all tests, significance was set at $P<0.05$. All statistical analysis used SAS software, v. 9.3.

\section{Results}

\section{Size}

Adenomas were smaller than non-adenomatous incidentalomas: $27.5 \pm 10.5 \mathrm{~mm} \quad($ range $=10-90 \mathrm{~mm}$ ) vs $63.6 \pm 35.1 \mathrm{~mm}$ (range: $15-200 \mathrm{~mm})(P<0.0001)$. The ROC curve for size had an AUC of 0.893 (CI=0.844-0.942) (Fig. 2A). The diagnostic performance of size for adenoma diagnosis is indicated in Table 1 . Ninety-two per cent of adenomas and $29 \%$ of non-adenomatous incidentalomas were $\leq 40 \mathrm{~mm}$. At this threshold, the sensitivity, specificity, PPV and NPV of CT were 91.8\% (CI=86.8-95.3), 71.4\% $(\mathrm{CI}=59.4-81.6), \quad 89.4 \% \quad(\mathrm{CI}=84.0-93.4) \quad$ and $\quad 76.9 \%$ $(\mathrm{CI}=64.8-86.5)$, respectively (Table 1$)$.
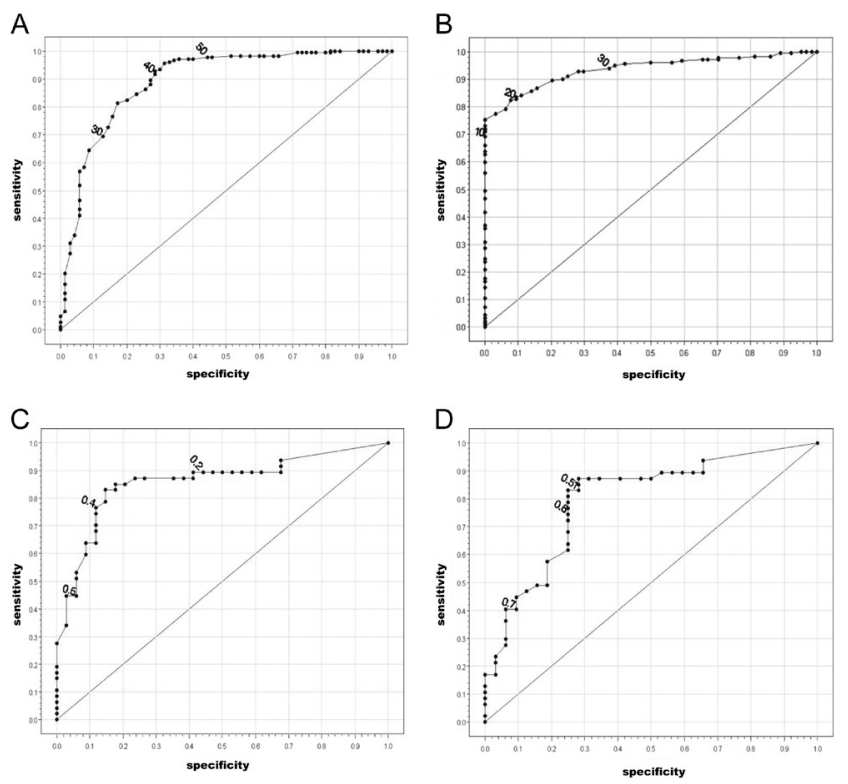

Figure 2

Receiving-operator curves for size $(A)$, unenhanced attenuation (B), relative percentage wash-out $(C)$ and absolute percentage wash-out (D).

\section{UA}

The UA was lower for adenomas than for nonadenomatous incidentalomas: $6.7 \pm 13.5 \mathrm{HU}$ (range: -27 to 45$)$ vs $33.3 \pm 8.6 \mathrm{HU}$ (range: $16-53)(P<0.0001)$. The ROC curve for UA had an AUC of 0.933 (CI=0.903-0.963) (Fig. 2). At a threshold of $10 \mathrm{HU}$, the sensitivity, specificity, PPV and NPV of CT for adenoma diagnosis were $69.2 \%$ $(\mathrm{CI}=62.0-75.8), 100 \%(\mathrm{CI}=94.4-100), 100 \%(\mathrm{CI}=97.1-$ $100)$ and 53.3\% $(\mathrm{CI}=44.0-62.5)$, respectively (Fig. 2B). A threshold of $15 \mathrm{HU}$ had $100 \%$ specificity, $100 \% \mathrm{PPV}$, $58.7 \% \mathrm{VPN}$ and $75.3 \%$ sensitivity.

\section{Parameter combinations}

The diagnostic performance of tumor-size and UA combinations at various thresholds is shown in Table 1. Combinations of size $\leq 30 \mathrm{~mm}+\mathrm{UA} \leq 20 \mathrm{HU}$ and size $\leq 40 \mathrm{~mm}+\mathrm{UA} \leq 15 \mathrm{HU}$, predicted the presence of an adenoma with $100 \%$ PPV.

\section{Relative percentage wash-out}

Eighty-two AIs without typical features of adenomas on unenhanced CT examination were examined with dynamic and delayed CT. The size and UA of these AIs were $38.6 \pm 24.1 \mathrm{~mm}$ (range: $14-11$ ) and $27.5 \pm 9.9 \mathrm{HU}$ (range: 12-53), respectively. They consisted of 47 adenomas (of which 24 were histologically confirmed) and 35 histologically confirmed non-adenomatous incidentalomas (18 pheochromocytomas, 12 other tumors including 8 ACC, 2 pseudocysts, 2 ganglioneuromas, 1 bilateral lymphoma, 1 angiosarcoma, 1 metastasis, 1 schwannoma). The mean RPW of the adenomas was higher than that of the non-adenomatous incidentalomas: $49 \% \pm 20 \%$ (range: $0-85$ ) vs $17 \% \pm 17 \%$ (range: $0-52$ ) $(P<0.0001)$. The ROC curve AUC for RPW was 0.862 ( $\mathrm{CI}=0.779-0.945$ ) (Fig. 2C). A 40\% threshold had a sensitivity, specificity, PPV and NPV for adenoma diagnosis of $76.6 \%(\mathrm{CI}=62.0-87.7), 88.6 \%(\mathrm{CI}=73.3-96.8), 90.0 \%$ $(\mathrm{CI}=76.3-97.2)$ and $73.8 \%(\mathrm{CI}=58.0-86.1)$, respectively. Perfect specificity was obtained with a threshold of $53 \%$ (Table 1).

\section{Absolute percentage wash-out}

The mean APW was higher for adenomas than for nonadenomatous incidentalomas: $62 \% \pm 22 \%$ (range: $0-95$ ) vs $30 \% \pm 27 \%$ (range: $0-77)(P<0.0001)$. The ROC curve AUC for APW was $0.802(\mathrm{CI}=0.701 ; 0.902)$ (Fig. 2D). 
Table 1 Accuracy of CT scanning for the diagnosis of adenoma.

\begin{tabular}{l} 
Threshold \\
\hline Size $(\mathrm{mm})$ \\
$\leq 30$ \\
$\leq 40$ \\
$\leq 50$ \\
UA $(\mathrm{HU})$ \\
$\leq 10$ \\
$\leq 15$ \\
$\leq 20$ \\
APW $(\%)$ \\
$>60$ \\
$>75$ \\
$>78$ \\
RPW $(\%)$ \\
$>40$ \\
$>50$ \\
$>53$ \\
Size + UA \\
$\leq 30+\leq 20$ \\
$\leq 40+\leq 15$ \\
$\leq 40+\leq 20$ \\
\hline
\end{tabular}

\begin{tabular}{c}
\hline Sensitivity (CI 95\%) \\
\hline $69.4(62.2-76.0)$ \\
$91.8(86.8-95.3)$ \\
$97.8(94.5-99.4)$ \\
\\
$69.2(62.0-75.8)$ \\
$75.3(68.3-81.4)$ \\
$83.0(76.6-88.1)$ \\
$72.3(57.4-84.4)$ \\
$23.4(12.3-38.0)$ \\
$17.0(7.6-30.8)$ \\
$76.6(62.0-87.7)$ \\
$44.7(30.2-59.9)$ \\
$27.7(15.6-42.6)$ \\
\\
$58.2(50.7-65.5)$ \\
$52.7(45.2-60.2)$ \\
$76.4(69.5-82.3)$
\end{tabular}

\begin{tabular}{c}
\hline Specificity $(\mathrm{Cl} 95 \%)$ \\
\hline $87.1(77.0-93.9)$ \\
$71.4(59.4-81.6)$ \\
$55.7(43.3-67.6)$ \\
\\
$100(94.4-100)$ \\
$100(94.4-100)$ \\
$90.6(80.7-96.5)$ \\
$75.8(57.7-88.9)$ \\
$97.0(84.2-99.9)$ \\
$100(89.4-100)$ \\
$88.6(73.3-96.8)$ \\
$94.3(80.8-99.3)$ \\
$100(90.0-100)$ \\
$100(94.4-100)$ \\
$100(94.4-100)$ \\
$96.9(89.2-99.6)$
\end{tabular}

\begin{tabular}{c}
\hline PPV (Cl 95\%) \\
\hline $93.4(87.8-96.9)$ \\
$89.4(84.0-93.4)$ \\
$85.2(79.7-89.7)$ \\
\\
$100(97.1-100)$ \\
$100(97.3-100)$ \\
$96.2(91.9-98.6)$ \\
\\
$81.0(65.9-91.4)$ \\
$91.7(61.5-99.8)$ \\
$100(63.1-100)$ \\
$90.0(76.3-97.2)$ \\
$91.3(72.0-98.9)$ \\
$100(75.3-100)$ \\
$100(96.6-100)$ \\
$100(96.2-100)$ \\
$98.6(95.0-99.8)$
\end{tabular}

NPV $(\mathrm{Cl}$ 95\%)

$52.1(42.7-61.5)$

$76.9(64.8-86.5)$

$90.7(77.9-97.4)$

$53.3(44.4-62.5)$

$58.7(48.9-68.1)$

$65.2(54.3-75.0)$

$65.8(48.6-80.4)$

$47.1(34.8-59.6)$

$45.8(34.0-58.0)$

$\mathrm{Cl}$, confidence interval; HU, Hounsfield units; NPV, negative predictive value; PPV, positive predictive value; UA, unenhanced attenuation.

A 60\% threshold had a sensitivity, specificity, PPV and NPV for adenoma diagnosis of $72.3 \%(\mathrm{CI}=57.4-84.4)$, 75.8\% (CI=57.7-88.9), 81.0\% (CI=65.9-91.4) and 65.8\% $(\mathrm{CI}=48.6-80.4)$, respectively. Perfect specificity was obtained with a threshold of 78\% (Table 1).

\section{Additional value of contrast wash-out studies}

Using our criterion for size and UA $(30 \mathrm{~mm}$ and $20 \mathrm{HU}$ or $40 \mathrm{~mm}$ and $15 \mathrm{HU}$, respectively) 16 of the 47 adenomas (34\%) that were subjected to contrast wash-out studies would have been correctly identified and would not therefore have required this additional CT investigation. Using the 53\% and 78\% thresholds for RPW and APW calculated in our study which also had a $100 \%$ specificity, $4(12.9 \%)$ of the 31 remaining atypical adenomas would have been correctly identified by contrast wash-out studies. Using the 'classical' 40\% and 60\% thresholds quoted in most studies for RPW and APW, 21 (67.7\%) of the 31 remaining atypical adenomas would have been correctly identified. Conversely, 5 pheochromocytomas and 2 pseudocysts would have been misdiagnosed as adenomas by contrast wash-out studies.

\section{Focus on pheochromocytomas and ACCs}

The 33 pheochromocytomas in this series measured $42.4 \pm 16.6 \mathrm{~mm} \quad$ (range: 15-80 $\mathrm{mm}$ ). Eighteen pheochromocytomas $(54 \%)$ were $\leq 40 \mathrm{~mm}$. UA was
$34.2 \pm 8.7 \mathrm{UH}$, and none of pheochromocytomas had a UA $\leq 15$ HU (range: $17-53$ ). Among the 18 pheochromocytomas analyzed by contrast wash-out studies; RPW was $24 \pm 18 \%$ and APW $36 \pm 27 \%$. RPW was $\geq 40 \%$ and APW $\geq 60 \%$ for respectively $22 \%$ and $29 \%$ of the pheochromocytomas.

The ACCs $(N=23)$ measured $91.0 \pm 41.5 \mathrm{~mm}$ (range: $37-200 \mathrm{~mm})$. Two ACCs $(8.7 \%$ of the study cohort) were $\leq 40 \mathrm{~mm}(37 \mathrm{~mm}$ and $38 \mathrm{~mm})$. UA was $35.0 \pm 7.9 \mathrm{UH}$ (range: 17-48) and no ACCs had a UA $\leq 15 \mathrm{HU}$. Among the 8 ACCs studied for contrast wash-out, RPW was $16 \pm 12 \%$ and APW $30 \pm 24 \%$. None of the adrenal carcinomas had an $\mathrm{RPW} \geq 40 \%$, and one had an $\mathrm{APW} \geq 60 \%$.

\section{Reproducibility of CT parameters}

The CCI, estimated in 197 patients, were $0.99(\mathrm{CI}=0.99$ 0.99), $0.96(\mathrm{CI}=0.94-0.97), 0.98(\mathrm{CI}=0.97-0.99)$ and 0.97 (CI=0.96-0.98) for size, UA, RPW and APW, respectively (Fig. 3).

\section{Discussion}

A recent thorough analysis of the literature shows that a stronger evidence base is needed to determine the optimal parameters of CT, and its performance, for the differential diagnosis of benign adenomas among AIs (3). A number of possible methodological biases have been identified in previous studies, including a referral pattern that may differ from that of the routine endocrinology setting. 

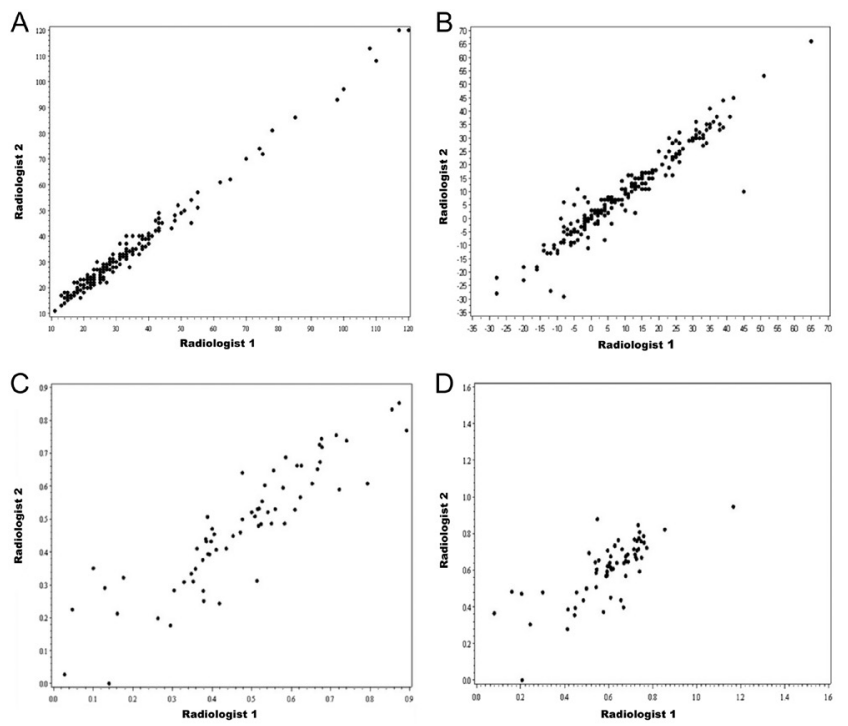

Figure 3

Reproducibility plots for size (A), unenhanced attenuation (B), relative percentage wash-out (C) and absolute percentage wash-out (D). The numbers shown in the figures correspond to different thresholds for CT parameters.

In our large study of well-characterized AIs detected in patients recruited in an endocrinological setting, we attempted to define the best CT parameters and thresholds to identify benign tumors, placing the accent on specificity, in order to avoid false diagnoses of benignity (the target condition being defined as adenomas and other benign tumors). Although sensitivity for adenomas is less critical, it needs to be high enough to avoid unnecessary investigations and surgery.

Size is often used to differentiate between adrenal adenomas and non-adenomatous incidentalomas and has been a major indication for surgery $(4,6$, $9,12,13)$. Our results confirm that, in patients with no known extra-adrenal malignancy, small AI size is suggestive of an adenoma. However, in our series, the classical size threshold of $40 \mathrm{~mm}$ would have missed 2 of 23 adrenocortical carcinomas (37 $\mathrm{mm}$ and $38 \mathrm{~mm}$ ) as well as half the pheochromocytomas and had a PPV for adenomas of $89.4 \%$. Smaller ACCs, less than $3 \mathrm{~cm}$, have already been reported $(7,12,13)$. It is noteworthy that the mean size of the incidentally discovered ACCs in our series was roughly the same as that observed in other series of ACCs with various presentations (6, $7,8,9,10)$. Intriguingly, and assuming that size is to some extent a function of time, our data suggest that incidental ACCs are not diagnosed at an earlier stage than non-incidental ACCs. Conversely, around 8\% of adenomas were above the $40-\mathrm{mm}$ size threshold. We thus confirm the large overlap between the size of incidentally discovered adenomas and that of other tumors, meaning that this criterion is rather inaccurate at the individual level.

Our study also confirms that a UA of $10 \mathrm{HU}$ is a diagnostically relevant threshold, a value below $10 \mathrm{HU}$ having a PPV of $100 \%(1,3)$ confirming the adenoma diagnosis. However UA $>10 \mathrm{UA}$, reflecting a low lipid content described in up to $43 \%$ of adrenal adenomas $(3,5,14,15,16,17)$ was found in a number of benign AIs in our series, resulting in a 53.3\% NPV. The NPV improved slightly, to $58.7 \%$, at a threshold of $15 \mathrm{UA}$, while maintaining a $100 \% \mathrm{PPV}$. This value is close to the $13 \mathrm{UH}$ threshold reported by Petersenn and coworkers, in the German ACCS registry (7), to exclude ACC with $100 \%$ specificity. We therefore confirm that incidentally discovered ACCs share the same basic CT characteristics as other ACCs.

Combinations of size $\leq 30 \mathrm{~mm}$ and UA $\leq 20 \mathrm{UA}$ and $\leq 40 \mathrm{~mm}$ and $\leq 15 \mathrm{UA}$, yielded a PPV of $100 \%$, a performance that is close to that found in a large surgical series of adrenal tumors (6). This combination of simple parameters could help to reduce the number of patients referred for complementary imaging or surgery, given that the number of benign AIs exceeds by far the number of ACCs (2). However, in clinical practice, this safe combination of size and UA may lack sensitivity for distinguishing adenomas from non-adenomatous incidentalomas $(<60 \%)$, meaning that complementary CT investigations may be required to characterize lipidpoor lesions.

Our analysis of inter-observer agreement, a feature that has seldom been reported $(7,17)$, revealed almost perfect agreement on both size and UA (CCI ranging from 0.96 to 0.99 ), suggesting that these thresholds may reliably be assessed by different operators.

Compared to adrenal adenomas, non-adenomatous adrenal tumors tend to retain, during a short period, a larger proportion of injected contrast material. This characteristic has represented the rationale for contrast wash-out studies. However, despite a number of publications suggesting the usefulness and good performance of this approach, only two studies were considered adequate for assessing with a minimum of bias the accuracy of wash-out tests for the characterization of lipid-poor AIs (3, 18, 19). Furthermore, the washout threshold that should be used to differentiate adenomatous from non-adenomatous adrenal tumors is controversial, and there are concerns that the sensitivity 
of this technique may be suboptimal. For example, in a small series of 11 patients with proven ACCs, 17 patients with proven pheochromocytomas and 23 patients with adrenal adenomas, Szolar et al. (9) found $100 \%$ sensitivity and specificity at threshold values of 50\% for APW and 40\% for RPW. By contrast, at the same thresholds, Sangwaiya et al. (16) reported a sensitivity of only $52 \%$ for both APW and RPW in a study of 323 adrenal adenomas. In another study, including 45 adenomas and 31 pheochromocytomas, Park et al. (20) found a specificity of only $61 \%$ at an APW of $60 \%$. Here, we analyzed the value of delayed wash-out, but only in AIs with equivocal UA findings. RWP and APW, at the classical respective thresholds of $40 \%$ and $60 \%$, correctly identified only $76.6 \%$ and $72.3 \%$ of 47 lipidpoor adenomas in our series. The specificity of RPW and APW in our series was also suboptimal (86.8\% and $75.6 \%$ respectively). However, none of the adrenal carcinomas were misdiagnosed using these wash criterion, a finding that is consistent the rarity of ACCs with rapid washout (14). On the contrary, pheochromocytomas were often misdiagnosed, as up to $29 \%$ of them displayed rapid wash-out. Park et al. (20) reported that 5 of 31 pheochromocytomas had an APW of $>60 \%$ and tended to be more hypervascular on pathological examination. This suggests that pheochromocytomas are the most common mimicker of adenomas in wash-out studies. In our series, the APW and RPW thresholds had to be raised to $78 \%$ and $53 \%$ respectively to ensure $100 \%$ specificity. At these thresholds, sensitivity for lipid-poor adrenal adenomas was markedly reduced. However, in clinical practice, this pitfall may be circumvented, as routine measurement of plasma or urinary metanephrines is recommended in the workup of AIs. Rare silent, incidentally discovered pheochromocytomas associated with normal plasma or urinary metanephrine values have been described, but none had ambiguous CT features (21). Therefore, the classical $60 \%$ and $40 \%$ thresholds for APW and RPW, although controversial (16), may safely be used as part of the standard AI workup. In our series, inter-observer differences in wash-out results were very small. A specific point of concern is the validity of our thresholds obtained using a 10-min delay during washout studies, for wash-out tests involving a 15-min delay. However, the systematic study by Korobkin et al. (22) addressed this issue and showed marginal differences in terms of thresholds, sensitivity and specificity between the 10- and 15-min delay wash-out protocols. As commonly recommended in clinical practice, caution should be exercised when interpreting borderline results. Elsewhere, our results could have been biased as only a subgroup of patients without typical features of adenomas on unenhanced CT examination were subjected to contrast wash-out studies.

Another weakness of this retrospective analysis is that 184 of 417 patients with AIs were excluded because they did not meet the one inclusion criteria about a follow-up long enough to ensure a better validity of the reference standard. Although this could have biased our findings, almost of these patients, who had little or no follow-up, had reassuring CT findings based on previous publications (5). Thus, the bias induced by their omission might result in an underestimation of the sensitivity of CT for detecting benign tumors. One strength of our series is that the definitive diagnoses were robust, being based on histology or imaging-based follow-up lasting at least one year while follow-up is usually limited to 6 months post studies (3). The other strengths of our study are the large number of consecutively selected patients with purely incidental findings, the endocrinological recruitment that included only a small number of patients with known extra-adrenal malignancies (as reflected by the presence of only one adrenal metastasis), the sufficient number of pheochromocytomas and ACCs needed to evaluate the specificity and PPV of CT, and the evaluation of interobserver agreement on the principal CT parameters in a large number of patients.

In conclusion, our study suggests that, in an endocrinological recruitment, combinations of CT criteria, namely size $\leq 40 \mathrm{~mm}$ and $\mathrm{UA} \leq 15 \mathrm{UH}$, and APW and RPW $>60 \%$ and $>40 \%$, respectively, are consistent with a diagnosis of adrenal adenoma and benign incidentaloma in patients with normal plasma or urinary metanephrines values. Other criteria, that were not considered here, such as irregular margins and rim enhancement, may also be useful for differential diagnosis of AIs, although neither of these imaging features has proven reliable for predicting malignancy (17). Further, prospective cohort studies with extended follow-up are needed to confirm our results and to determine the most efficient and cost-effective imaging strategy for AIs with uncertain diagnosis at CT (1).

Declaration of interest

The authors declare that there is no conflict of interest that could be perceived as prejudicing the impartiality of this study.

Funding

This research did not receive any specific grant from any funding agency in the public, commercial or not-for-profit sector. 


\section{Acknowledgements}

The authors are grateful to Anjarasoa Tsaranazy for her work in the statistical analysis of the data and to Amandine Galioot for editorial assistance.

\section{References}

1 Fassnacht M, Arlt W, Bancos I, Dralle H, Newell-Price J, Sahdev A, Tabarin A, Terzolo M, Tsagarakis S \& Dekkers OM. Management of adrenal incidentalomas: European Society of Endocrinology Clinical Practice Guideline in collaboration with the European Network for the study of adrenal tumors. European Journal of Endocrinology 2016 175 G1-G34. (https://doi.org/10.1530/EJE-16-0467)

2 Cawood TJ, Hunt PJ, O’Shea D, Cole D \& Soule S. Recommended evaluation of adrenal incidentalomas is costly, has high false-positive rates and confers a risk of fatal cancer that is similar to the risk of the adrenal lesion becoming malignant; time for a rethink? European Journal of Endocrinology 2009161 513-527. (https://doi.org/10.1530/ EJE-09-0234)

3 Dinnes J, Bancos I, Ferrante di Ruffano L, Chortis V, Davenport C, Bayliss S, Sahdev A, Guest P, Fassnacht M, Deeks JJ et al. Management of endocrine disease: imaging for the diagnosis of malignancy in incidentally discovered adrenal masses: a systematic review and meta-analysis. European Journal of Endocrinology 2016175 R51-R64. (https://doi.org/10.1530/EJE-16-0461)

4 Terzolo M, Stigliano A, Chiodini I, Loli P, Furlani L, Arnaldi G, Reimondo G, Pia A, Toscano V, Zini M et al. AME position statement on adrenal incidentaloma. European Journal of Endocrinology 2011164 851-870. (https://doi.org/10.1530/EJE-10-1147)

5 Boland GW. Adrenal imaging: why, when, what, and how? Part 3. The algorithmic approach to definitive characterization of the adrenal incidentaloma. American Journal of Roentgenology 2011196 W109-W111. (https://doi.org/10.2214/AJR.10.4206)

6 Hamrahian AH, Ioachimescu AG, Remer EM, Motta-Ramirez G, Bogabathina H, Levin HS, Reddy S, Gill IS, Siperstein A \& Bravo EL. Clinical utility of noncontrast computed tomography attenuation value (Hounsfield units) to differentiate adrenal adenomas/ hyperplasias from nonadenomas: Cleveland Clinic experience. Journal of Clinical Endocrinology and Metabolism 200590 871-877. (https://doi.org/10.1210/jc.2004-1627)

7 Petersenn S, Richter PA, Broemel T, Ritter CO, Deutschbein T, Beil FU, Allolio B \& Fassnacht M. Computed tomography criteria for discrimination of adrenal adenomas and adrenocortical carcinomas: analysis of the German ACC registry. European Journal of Endocrinology 2015172 415-422. (https://doi.org/10.1530/EJE-14-0916)

8 Slattery JM, Blake MA, Kalra MK, Misdraji J, Sweeney AT, Copeland PM, Mueller PR \& Boland GW. Adrenocortical carcinoma: contrast washout characteristics on CT. American Journal of Roentgenology $2006 \mathbf{1 8 7}$ W21-W24. (https://doi.org/10.2214/AJR.04.1751)

9 Szolar DH, Korobkin M, Reittner P, Berghold A, Bauernhofer T, Trummer H, Schoellnast H, Preidler KW \& Samonigg H. Adrenocortical carcinomas and adrenal pheochromocytomas: mass and enhancement loss evaluation at delayed contrast-enhanced CT. Radiology 2005234 479-485. (https://doi.org/10.1148/ radiol.2342031876)

10 Abiven G, Coste J, Groussin L, Anract P, Tissier F, Legmann P, Dousset B, Bertagna X \& Bertherat J. Clinical and biological features in the prognosis of adrenocortical cancer: poor outcome of cortisol-secreting tumors in a series of 202 consecutive patients. Journal of Clinical Endocrinology and Metabolism 200691 2650-2655. (https://doi.org/10.1210/jc.2005-2730)

11 Tabarin A, Bardet S, Bertherat J, Dupas B, Chabre O, Hamoir E, Laurent F, Tenenbaum F, Cazalda M, Lefebvre H et al. Exploration and management of adrenal incidentalomas. French Society of Endocrinology Consensus. Annales D'Endocrinologie 200869 487-500. (https://doi.org/10.1016/j.ando.2008.09.003)

12 Mansmann G, Lau J, Balk E, Rothberg M, Miyachi Y \& Bornstein SR. The clinically inapparent adrenal mass: update in diagnosis and management. Endocrine Reviews 200425 309-340. (https://doi. org/10.1210/er.2002-0031)

13 Mantero F, Terzolo M, Arnaldi G, Osella G, Masini AM, Ali A, Giovagnetti M, Opocher G \& Angeli A. A survey on adrenal incidentaloma in Italy. Study Group on Adrenal Tumors of the Italian Society of Endocrinology. Journal of Clinical Endocrinology and Metabolism 200085 637-644.

14 Caoili EM, Korobkin M, Francis IR, Cohan RH, Platt JF, Dunnick NR \& Raghupathi KI. Adrenal masses: characterization with combined unenhanced and delayed enhanced CT. Radiology 2002222 629-633. (https://doi.org/10.1148/radiol.2223010766)

15 Pena CS, Boland GW, Hahn PF, Lee MJ \& Mueller PR. Characterization of indeterminate (lipid-poor) adrenal masses: use of washout characteristics at contrast-enhanced CT. Radiology $20002 \mathbf{2 1 7}$ 798-802. (https://doi.org/10.1148/radiology.217.3.r00dc29798)

16 Sangwaiya MJ, Boland GW, Cronin CG, Blake MA, Halpern EF \& Hahn PF. Incidental adrenal lesions: accuracy of characterization with contrast-enhanced washout multidetector CT - 10-minute delayed imaging protocol revisited in a large patient cohort. Radiology 2010256 504-510. (https://doi.org/10.1148/ radiol.10091386)

17 Song JH, Grand DJ, Beland MD, Chang KJ, Machan JT \& Mayo-Smith WW. Morphologic features of 211 adrenal masses at initial contrast-enhanced CT: can we differentiate benign from malignant lesions using imaging features alone? American Journal of Roentgenology 2013201 1248-1253. (https://doi.org/10.2214/AJR.12.10302)

18 Angelelli G, Mancini ME, Moschetta M, Pedote P, Pignataro P \& Scardapane A. MDCT in the differentiation of adrenal masses: comparison between different scan delays for the evaluation of intralesional washout. Scientific World Journal 20132013957680.

19 Vilar L, Freitas Mda C, Canadas V, Albuquerque JL, Botelho CA, Egito CS, Arruda MJ, Moura e Silva L, Coelho CE, Casulari LA et al. Adrenal incidentalomas: diagnostic evaluation and longterm follow-up. Endocrine Practices 200814 269-278. (https://doi. org/10.4158/EP.14.3.269)

20 Park BK, Kim B, Ko K, Jeong SY \& Kwon GY. Adrenal masses falsely diagnosed as adenomas on unenhanced and delayed contrastenhanced computed tomography: pathological correlation. European Radiology 200616 642-647. (https://doi.org/10.1007/ s00330-005-0017-0)

21 Haissaguerre M, Courel M, Caron P, Denost S, Dubessy C, Gosse P, Appavoupoulle V, Belleannee G, Jullie ML, Montero-Hadjadje M et al. Normotensive incidentally discovered pheochromocytomas display specific biochemical, cellular, and molecular characteristics. Journal of Clinical Endocrinology and Metabolism 201398 4346-4354. (https:// doi.org/10.1210/jc.2013-1844)

22 Korobkin M, Brodeur FJ, Francis IR, Quint LE, Dunnick NR \& Londy F. CT time-attenuation washout curves of adrenal adenomas and nonadenomas. American Journal of Roentgenology $1998 \mathbf{1 7 0}$ 747-752. (https://doi.org/10.2214/ajr.170.3.9490968)
Received 2 January 2018

Revised version received 13 February 2018

Accepted 20 February 2018 